\title{
Are Cancer Patients Willing to Travel More or Further Away for a Slightly More Efficient Therapy?
}

\author{
Ph. Groux ${ }^{1}$, S. Anchisi ${ }^{2} \&$ Th. Szucs ${ }^{3}$ \\ ${ }^{1}$ Kundengerecht.ch GmbH, Alpenstrasse 22, Huttwil 4950, Switzerland \\ ${ }^{2}$ Département de médecine interne et gériatrie, service d'oncologie, CHVR-hôpital du Valais, Avenue de \\ Grand-Champsec 80, Sion 1951, Switzerland \\ ${ }^{3}$ European Center of Pharmaceutical Medicine, University of Basel, Klingelbergstrasse 61, Basel 4056, \\ Switzerland
}

Correspondence: Dr. Philippe Groux, MPH, Kundengerecht.ch GmbH, Alpenstrasse 22, Huttwil 4950, Switzerland. Tel: 41-79-203-1411. E-mail: philippe.groux@kundengerecht.ch

Received: May 1, 2014 Accepted: May 25, 2014 Online Published: May 27, 2013

doi:10.5539/cco.v3n1p36 URL: http://dx.doi.org/10.5539/cco.v3n1p36

\begin{abstract}
Centralisation of cancer services is ongoing in many countries due to the rapid increase of knowledge in oncology, the interdisciplinary of many cancer treatments and economic pressure. This study investigated whether cancer patients are willing to accept this additional burden. All patients who came in February 2012 for a consultation or an ambulant treatment were asked to answer to a survey. The questionnaire covered among other six questions whether the patients would be willing to travel more frequently or further away. These questions were split in two groups: one for a slightly more efficacious therapy and the other for a therapy with slightly fewer side effects. To travel more frequently is well accepted as a clear majority of patients say yes or rather yes to travel more frequently for a more efficacious therapy $(67 \%)$ as for a therapy with fewer side effects $(62 \%)$. To travel further away doesn't have a clear majority as $51 \%$ of the patients say yes or rather yes for a more efficacious therapy and $49 \%$ for a therapy with fewer side effects. General acceptance of travelling further away as a consequence of centralisation of oncology services cannot be assumed. Particularly elderly patients are reluctant to it and will require specific solutions.
\end{abstract}

Keywords: access to care, elderly patients, burden of disease, therapy efficiency

\section{Background}

Centralisation of cancer services is ongoing in many countries due to the rapid increase of knowledge in oncology and the interdisciplinary of many cancer treatments and due to economic pressure. Nevertheless evidence that centralisation of cancer services lead to cost-effective care is incomplete (Ke et al., 2012). The need to travel for treatment cause many practical, emotional and financial problems for patients and burden them with additional worry concerning family and work commitments (Butow et al., 2012). Recent publications highlighted as well the travel-related burden for cancer patients (Zucca et al., 2011) as the financial and social impact for support persons of cancer survivors (Carey et al., 2012). Use of the less travelling intensive hypofractionated breast radiotherapy versus conventional breast radiotherapy was recently published as strongly correlated with the distance to the clinic (Page et al., 2013). Some reports reveal that an average of 20-30\% of North American women forego radiotherapy after receiving lumpectomy (Chuba \& Simon, 1997; Malin, Schuster, Kahn, \& Brook, 2002), or choose mastectomy over breast preservation (Tuttle, Habermann, Grund, Morris, \& Virnig, 2007) due to several factors, including age, psychological distress, quality of life concerns, and cost of undergoing prolonged daily radiotherapy (Dwyer, Hickey, Burmeister, \& Burmeister, 2010).

The Swiss health system is based on principles of free demand and supply as well as regulated competition (Busato \& Künzi, 2008). Most services are reimbursed on a fee-for-service system; nevertheless travel costs are not reimbursed. Chemotherapy is provided as well by oncology wards in hospitals and as by private practices of medical oncologists and haematologists. Only physicians with dedicated certificates of competence are allowed to prescribe chemotherapies, in fact almost only medical oncologists and haematologists have these certificates.

Valais is a Swiss alpine canton at the southern border of the country and consists in a main valley and the valleys 
of several feeder rivers. The population of the upper part of the valley speaks German, the population in the middle and the lower part of the valley speaks French. All cancer treatments are offered in cities in the main valley. Chemotherapies are offered by the Hôpital du Valais in four different regional sites (Brig, Martigny, Sion, Sierre), by the Hôpital du Chablais-Riviera in Monthey and by a private practice in Sierre. Eighty-six per cent of the population has access to chemotherapy within a 30 minute drive. A radio-oncology service is offered in Sion. A former study emphasized transport issues as a major obstacle to cancer treatment (S. Anchisi \& A. Anchisi, 2008). A recent study demonstrated that cancer patients in Valais outline this issue when discussing cancer treatment as well with their general practitioner as with their medical oncologist (Groux \& Szucs, 2013).

We investigated whether all patients who came in February 2012 for consultation or ambulant therapy to one of the four centres of the Hôpital du Valais or the private practice would theoretically accept to travel more frequently or further away for a slightly more efficient therapy. The aim was to characterize and quantify the acceptance to travel further away and to identify groups of patients who probably need particular solutions.

\section{Methods}

All patients who came in February 2012 for consultation or ambulant treatment to one of the four centres of the Hôpital du Valais or to the private practice were asked to answer a survey. Exception was made with patients who came for the first time and didn't have a diagnosis yet. All patients were asked at least once per centre to fill out the questionnaire. Patients who were asked twice or more were free to answer again or not. If a patient came up in two different centres he was asked to answer in both centres. The questionnaire covered several themes as age, kind of cancer, kind of treatment, how the patient travelled to the centre, how long the travel lasted and six questions whether the patients would be willing to travel more frequently or further away. These questions were split in two groups: one for a slightly more efficacious therapy and the other for a therapy with slightly fewer side effects.

The first group of questions was "if a slightly more efficacious therapy as your actual therapy would exist, would you be willing to (a) travel more frequently, (b) travel further away and (c) be treated in another canton?" with the following options to choose (1) No, (2) rather no, (3) rather yes, (4) yes and (5) I don't know.

The second group of questions was "if a therapy with slightly fewer side effects as your actual therapy would exist, would you be willing to (a) travel more frequently, (b) travel further away and (c) be treated in another canton?" with the following options to choose (1) No, (2) rather no, (3) rather yes, (4) yes and (5) I don't know.

The data were summarized and analyzed using Microsoft Excel and EpiData Analysis (Epidata Association, Odense, Denmark). Patients with contradictory answers, not living in Valais or not having cancer were excluded. If a patient answered twice to the questionnaire at the same site only the first questionnaire was taken in consideration.

The four distance groups (see Table 1) were not homogenous for the language ratio (20\% German speaking patients in group $1,26 \%$ in group $2,55 \%$ in group 3, $84 \%$ in group 4). Language is important because the distance to the next possible site is longer for the German speaking patients as a trip from the unique oncology ward in the German speaking part (Brig) to the secondary hospital (Sion) lasts 60 minutes while a trip from an oncology ward in the French speaking part to the secondary hospital lasts only 15 to 30 minutes. Differences in median age (61 years for group 1, 62 years for group 2, 65 years for group 3 and 65 years for group 4) were minor. To allow comparisons between distance group data were standardized for language and age. Approval for the study was obtained from the medical-ethical commission of the canton of Valais.

\section{Results}

629 questionnaires were handed out from which 619 were collected. 408 different patients participated to the survey. $323(79 \%)$ different patients answered to all requested questions for this publication $(79 \%$ of all participating patients). The patient characteristics are summarized in Table 1, the answers in Table 2. 
Table 1. Patient characteristics

\begin{tabular}{cc}
\hline Patient characteristics & Result \\
\hline Gender & $178(55 \%)$ \\
Women & $145(45 \%)$ \\
Men & 63 years \\
Median age & \\
Age & 19 years old \\
Youngest patient & 87 years old \\
Oldest patient & \\
Language & $218(67 \%)$ \\
French & 105 German $(33 \%)$ \\
German & $130(40 \%)$ \\
Distance to treatment centre & $112(35 \%)$ \\
Below 15 minutes & $62(19 \%)$ \\
Between 15 and 30 minutes & $19(6 \%)$ \\
Between 30 and 60 minutes & \\
More than 60 minutes &
\end{tabular}

Table 2. Answers of all 323 patients to the question

\begin{tabular}{lcc}
\hline \multicolumn{1}{c}{ Question } & $\begin{array}{c}\text { Answer for a slightly more } \\
\text { efficacious therapy }\end{array}$ & $\begin{array}{c}\text { Answer for a therapy with } \\
\text { slightly fewer side effects }\end{array}$ \\
\hline $\begin{array}{l}\text { Would you be willing to travel } \\
\text { more frequently }\end{array}$ & & \\
No & $10(3 \%)$ & $19(6 \%)$ \\
Rather No & $27(8 \%)$ & $18(6 \%)$ \\
Rather Yes & $54(17 \%)$ & $48(15 \%)$ \\
Yes & $161(50 \%)$ & $152(47 \%)$ \\
I don't know & $38(12 \%)$ & $51(16 \%)$ \\
No answer & $23(10 \%) r$ & $35(11 \%)$ \\
Would you be willing to travel & & \\
further away & & $22(7 \%)$ \\
No & $18(6 \%)$ & $28(9 \%)$ \\
Rather No & $32(10 \%)$ & $43(13 \%)$ \\
Rather Yes & $37(11 \%)$ & $115(36 \%)$ \\
Yes & $130(40 \%)$ & $28(9 \%)$ \\
I don't know & $25(8 \%)$ & $87(27 \%)$ \\
No answer & $81(25 \%)$ & \\
Would you be willing to be & & $27(8 \%)$ \\
treated in another canton & & $36(11 \%)$ \\
No & $22(7 \%)$ & $35(11 \%)$ \\
Rather No & $40(12 \%)$ & $112(35 \%)$ \\
Rather Yes & $37(12 \%)$ & $32(10 \%)$ \\
Yes & $122(38 \%)$ & $81(25 \%)$ \\
I don't know & $25(8 \%)$ & \\
No answer & $77(24 \%)$ & \\
\hline
\end{tabular}

To travel more frequently is well accepted, as a clear majority of patients say yes or rather yes as well to travel more for a more efficacious therapy (67\%) as for a therapy with fewer side effects (62\%). Only $46(16 \%)$ 
patients are reluctant or refuse to travel more frequently at least for one of both reasons proposed.

If the positive attitude group is homogenous as $93 \%$ of the patients who said yes or rather yes to the question with "more efficacious" answered also yes to the question with "fewer side effects", this is not the case within the negative attitude group as $44 \%$ of the patients with a negative attitude to the more efficacious therapy were positive for the therapy with fewer side effects. Only 18 patients $(6 \%)$ answer no or rather no to both questions.

To travel further away doesn't have a clear majority as $51 \%$ of the patients say yes to travel further away for a more efficacious therapy and $49 \%$ for a therapy with fewer side effects. $89 \%$ of the patients being positive to travel further for a more efficacious therapy are also positive for a therapy with fewer side effects. Only 60 (19\%) patients are negative to travel further away for at least one reason offered. The homogeneity for negative attitude is with clearly higher than for travelling more frequently as 32 patients $(10 \%)$ say no or rather no to both questions.

The idea to be treated in another canton is still accepted by $50 \%$ of the patients for a more efficacious therapy and $46 \%$ for a treatment with fewer side effects. $40 \%$ of the patients say yes or rather yes to both questions. $15 \%$ say no or rather no to both questions. The probability to get a double positive answer (91\%) or a double negative answer (88\%) is high.

We analysed whether acceptance is influenced by gender, age and distance to the actual treatment place. If no statistically significant difference can be found for gender, age highly impacts the acceptance (see Figure 1).

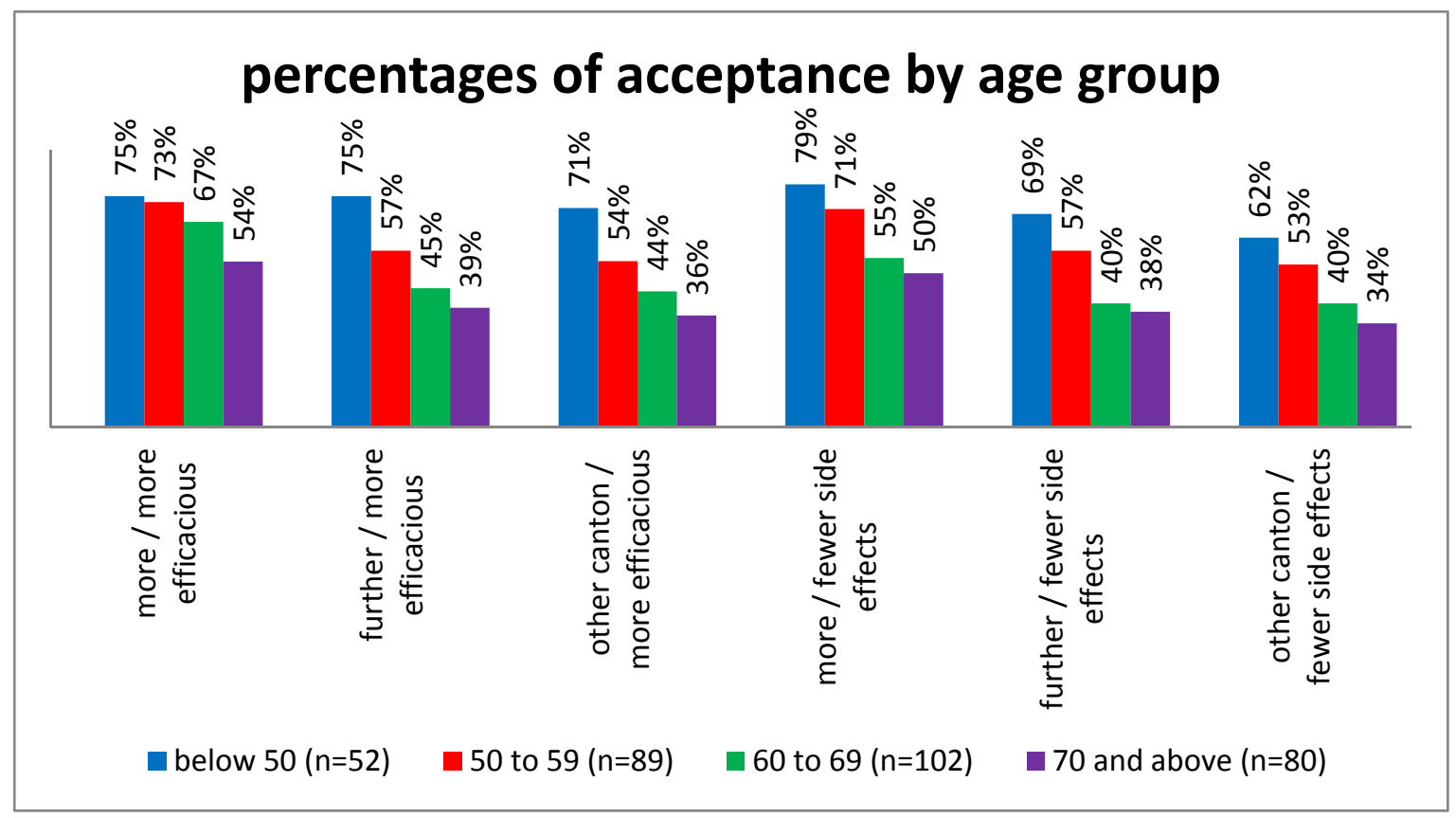

Figure 1. Answers by age groups

Acceptance declines with age. To travel more frequently is accepted by all age groups for a more efficacious therapy, nevertheless the probability of a refusal is statistically significantly higher for patients with an age of 70 years or more compared to patients below 70 years (OR 2.7, 95\% CI: 1.3 - 5.7). The acceptance to travel more for a therapy with slightly fewer side effects drops at 60 years and more (OR 2.9, 95\% CI: 1.3 - 6.4), remains nevertheless positive for a small majority of patients between 60 and 69 whilst patients of 70 and above being positive to travel more are as numerous as those negative to it. The interrelationship between acceptance and age is reflected by the median age: patients positive to both options have a median age of 58 years, patients positive for a therapy with less side effects but not for a more efficacious one have a median age of 60 years, patients positive for a more efficacious therapy but not for one with less side effects have a median age of 66 years. 66 years is also the median age of patients reluctant to both options.

To travel further away finds a majority of acceptance in patients below 60 but not in patients older than 60 , regardless whether the question was just "further away" or "being treated in another canton". Median age of 
patients positive to both options is 57 years; median age of patients reluctant to one or both options is 65 years with no difference in median age whether the patient is only reluctant to travel further away for a more efficacious therapy, only reluctant for a therapy with fewer side effects or reluctant to both options. Patients positive to both options for a treatment in another canton have a median age of 58 years, patients positive for a more efficacious therapy but not for a therapy with fewer side effects have a median age of 59 years, patients positive for a therapy with fewer side effects but not for a more efficacious therapy have a median age of 63 years and patients reluctant to both options have a median age of 66 years.

The perception of travelling further away is influenced by how much further away this would be compared to the actual journey. To allow comparison among distance groups the results are therefore adjusted as well for age as for language (Figure 2).

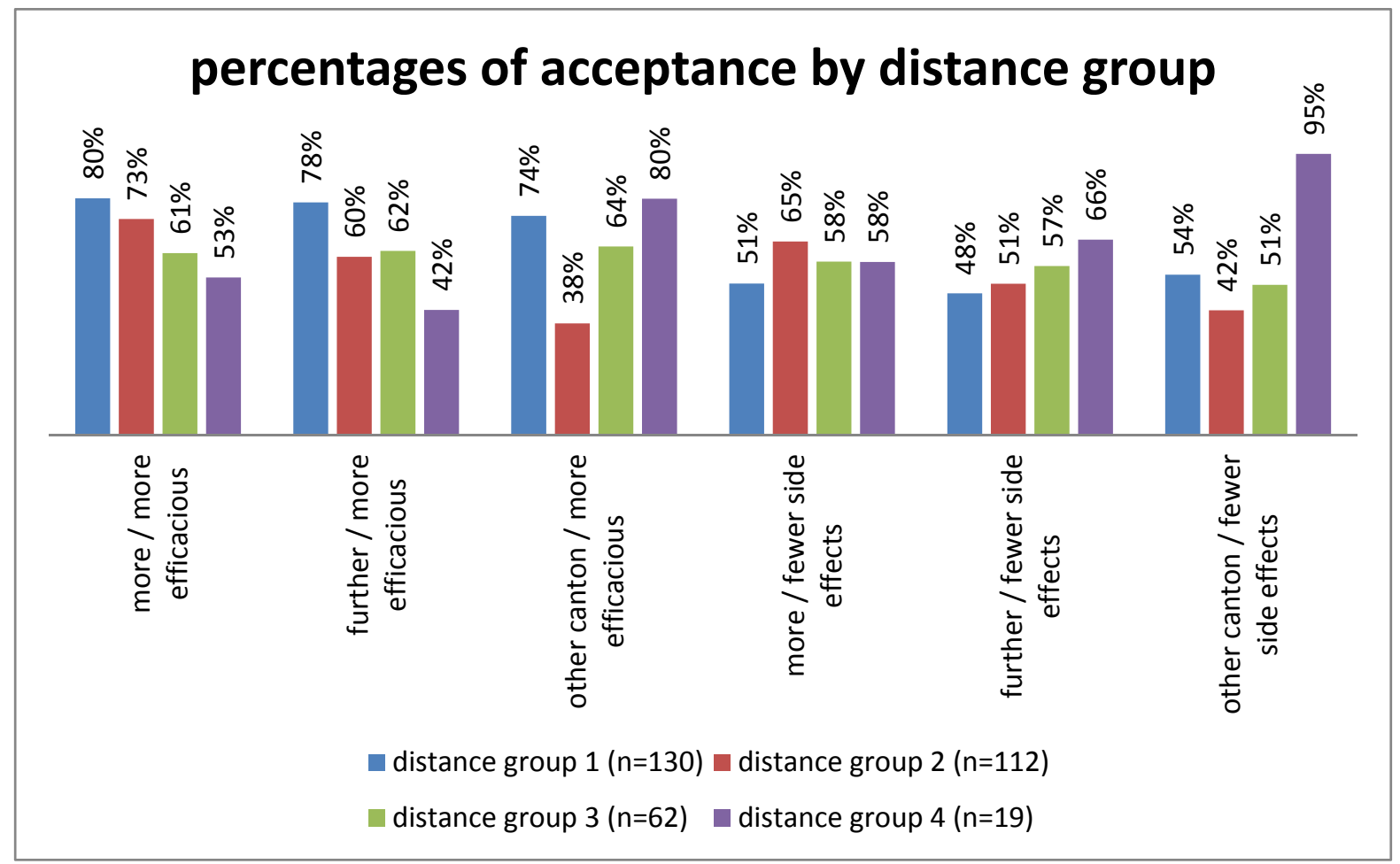

Figure 2. Age-language adjusted results for the distance groups. Distance group 1 equals to less than 15 minutes for one way, group 2 to 15 to 30 minutes, group 3 to 30 to 60 minutes and group 4 to more than 60 minutes

Acceptance to travel more for a more efficacious therapy rapidly declines with distance whilst distance doesn't impact on the acceptance to travel more for a therapy with fewer side effects. If acceptance to travel further away for a more efficacious therapy declines with distance (from $78 \%$ for distance group 1 to $42 \%$ for distance group 4) it increases with distance for a therapy with fewer side effects (from $48 \%$ for distance group 1 to $66 \%$ for distance group 4). To be treated in another canton is highly accepted by patients of distance group 4 ( $80 \%$ resp. $95 \%$ for a more efficacious therapy or a therapy with fewer side effects). For distance group 1 the acceptance is different whether the treatment would be a more efficacious one or one with fewer side effects, similarly to the acceptance to travel more.

\section{Discussion}

The willingness to travel more frequently or further away for a slightly more effective therapy is impacted by age and distance, whether the increased travel activity would be more journeys to the same place or journeys to another place and whether the higher effectiveness offered was more efficacy or fewer side effects.

Age is the major impact factor. Younger patients are more open for more or further travelling. Whether this in linked to a higher mobility, to hope of a higher impact in life expectancy, less metastatic diseases or some other factors cannot be answered with our data. The acceptance drops dramatically with age. If to travel more is still accepted by a small majority of elder patients ( 70 years and above), to travel further away or being treated in 
another canton is refused by a majority of elder patients.

To be treated in another canton is highly accepted by patients already having a long journey (more than an hour). We assume that the reasons are different for French speaking and German speaking patients. French speaking patients with such a long journey live close to the French speaking canton of Vaud and get their actual treatment in Sion because it is not available in a closer oncology ward, mainly radiotherapy. The trip to a hospital in the canton Vaud including the university hospital in Lausanne wouldn't last longer than the trip to Sion. As radio oncology patients are in general younger than chemotherapy the influence of age described above impacts favourably the willingness to be treated in another canton. For German speaking patients the trip in another canton would be a treatment in a hospital in the German speaking canton of Berne as alternative to the French speaking hospitals in Valais. The potentially greater distance, depending in which hospital in the canton of Berne they would be treated, could be over weighted by the barrier of language.

Whether a higher efficiency was proposed as more efficacy or as fewer side effects wasn't perceived as equal solutions by all patients, particularly when the option was to travel more. If acceptance to travel more for a more efficacious therapy lead very frequently also to acceptance to travel more for fewer side effects, refusal of more travelling for more efficacy wasn't linked to refusal of more travelling for fewer side effects and vice-versa. For patients living close to the oncology ward (less than 15 minutes) a more efficacious therapy was more attractive than a therapy with fewer side effects when the option was to travel more. This gap disappeared with increasing distance. When the option was to travel further away acceptance was more favourable for fewer side effects than for more efficacy for the patients having a long journey (more than an hour). Whether these differences in acceptance are linked to an increased burden by the journey the longer the journey is should be investigated. The difference in acceptance in distance group 1 with a better acceptance for travelling more frequently for a more efficacious therapy but not for a therapy with fewer side effects could be linked to an increased burden by travelling which over weights the reduced burden by side effects of the therapy.

The study methodology has some limitations, particularly the voluntariness as well to participate to the survey as to answer to the specific questions of interest for this investigation. Only 207 patients (64\%) answered to all these questions and 1/3 questions get no answer or a "don't know" answer. Furthermore the wording of the questions with the imprecise options "more" and "further" lets room for interpretation and does not allow to say to which extend patients are willing to increase the frequency and the distance of travelling. The options "slightly more efficacious therapy" and "therapy with slightly fewer side effects" allow as well room for interpretation. Whether the patient was willing to travel more was answered by $90 \%$ (more efficacy) resp. $89 \%$ of the patients (fewer side effects) whilst the percentage dropped to $75 \% / 73 \%$ for "travelling further away" and $76 \% / 75 \%$ "being treated in another canton". The first question seemed easy to answer whilst the second and third caused difficulties. Nevertheless the percentage of patients answering "I don't know" is lower in question two and three and leads to a percentage of exploitable answers which is only slightly lower than for the first question.

\section{Conclusion}

The willingness to travel more or further away for a slightly more effective therapy is different whether the increased travel activity would be more journeys to the same place or journeys to another place and whether the higher effectiveness is more efficacy or fewer side effects. Age is the major factor with impact on the willingness with a decrease of willingness with increasing age. Distance impacts as well on the willingness, nevertheless with different consequences whether on one hand the option was to travel more, further away or to be treated in another canton and on the other hand how much the journey to the actual treatment ward lasted.

General acceptance of travelling further away as a consequence of centralisation of oncology services cannot be assumed. The difference of acceptance of travelling more to the same place or travelling further away demonstrate that acceptance is not linked to additional distance per se but to overcome greater global burden of the therapy, travelling being one of the many side effects of these treatments. Particularly elderly patients are reluctant to it and will require specific solutions. Our observational study can help to generate hypothesis for additional research is necessary to understand the reasons for the different reluctances described.

Further analysis of our data is ongoing and will be published separately.

\section{Acknowledgements}

The authors would like to gratefully acknowledge the staff of the five involved centres for their active role by encouraging patients to fill out the questionnaire. This study has been supported by an unrestricted grant from Pierre Fabre Oncology Switzerland. 


\section{References}

Anchisi, S., \& Anchisi, A. (2008). Vivre la chimiothérapie au quotidien: un processus qui révèle l'âge. Bull Cancer, 95(suppl. FMC), F44-50.

Busato, A., \& Künzi, B. (2008). Primary care physician supply and other key determinants of health care utilization: the case of Switzerland. BMC Health Services Research, 8(8). http://dx.doi.org/10.1186/1472-6963-8-8

Butow, P. N., Phillips, F., Schweder, J., White, K., Underhill, C., \& Goldstein, D. (2012). Psychosocial well-being and supportive care needs of cancer patients living in urban and rural/regional areas: a systematic review. Supportive Care in Cancer, 20(1), 1-22. http://dx.doi.org/10.1007/s00520-011-1270-1

Carey, M., Paul, C., Cameron, E., Lynagh, M., Hall, A., \& Tzelepis, F. (2012). Financial and social impact of supporting a haematological cancer survivor. European journal of cancer care, 21(2), 169-176. http://dx.doi.org/10.1111/j.1365-2354.2011.01302.x

Chuba, P. J., \& Simon, M. S. (1997). Trends in primary surgical and radiation therapy for localized breast cancer in the Detroit Metropolitan area 1973-1992. International Journal of Radiation Oncology* Biology* Physics, 38(1), 103-107. http://dx.doi.org/10.1016/S0360-3016(96)00615-3

Dwyer, P., Hickey, B., Burmeister, E., \& Burmeister, B. (2010). Hypofractionated whole-breast radiotherapy: impact on departmental waiting times and cost. $J$ Med Imaging Radiat Oncol, 54(3), 229-234. http://dx.doi.org/10.1111/j.1754-9485.2010.02163.x

Groux, P., \& Szucs, T. (2013). Geographic disparities in access to cancer care: do patients in outlying areas talk about their access problems to their general practitioners and medical oncologists and how does that impact on the choice of chemotherapy?. European journal of cancer care, 22(6), 746-753. http://dx.doi.org/10.1111/ecc.12096

Ke, K. M., Hollingworth, W., \& Ness, A. R. (2012). The costs of centralisation: a systematic review of the economic impact of the centralisation of cancer services. European journal of cancer care, 21(2), 158-168. http://dx.doi.org/10.1111/j.1365-2354.2011.01323.x

Malin, J. L., Schuster, M. A., Kahn, K. A., \& Brook, R. H. (2002). Quality of breast cancer care: what do we know?. J Clin Oncol, 20(21), 4381-4393. http://dx.doi.org/10.1200/JCO.2002.04.020

Page, B. R., Belnap, T., Christopher Bowen, R., Gaffney, D. K., \& Sause, W. T. (2013). Utilization of Hypofractionated and Conventional Breast Radiotherapy in the State of Utah. Cancer \& Clinical Oncology, 2(2). http://dx.doi.org/10.5539/cco.v2n2p34

Tuttle, T. M., Habermann, E. B., Grund, E. H., Morris, T. J., \& Virnig, B. A. (2007). Increasing use of contralateral prophylactic mastectomy for breast cancer patients: a trend toward more aggressive surgical treatment. J Clin Oncol, 25(33), 5203-5209. http://dx.doi.org/10.1200/JCO.2007.12.3141

Zucca, A., Boyes, A., Newling, G., Hall, A., \& Girgis, A. (2011). Travelling all over the countryside: Travel-related burden and financial difficulties reported by cancer patients in New South Wales and Victoria. Aust. J. Rural Health, 19, 298-305. http://dx.doi.org/10.1111/j.1440-1584.2011.01232.x

\section{Copyrights}

Copyright for this article is retained by the author(s), with first publication rights granted to the journal.

This is an open-access article distributed under the terms and conditions of the Creative Commons Attribution license (http://creativecommons.org/licenses/by/3.0/). 